\title{
Research and Implementation of Virtual Building Roaming System Based on VRML
}

\author{
Lin Zhang ${ }^{\mathrm{i}}$ \\ School of Electricity and Information Engineering \\ BUCEA \\ Beijing, 100044, China \\ e-mail: zzcmeng0806@sina.com
}

\begin{abstract}
Research and implementation of virtual building roaming is a new technology rised recently.It can use 3D modeling tools, VRML tools and X3D browser software to complete the roamming system.This paper introduces the development method,technology and process,and takes the Fragrant Hill Park for example, completes the virtual roamming system. The system is realistic and provides users proper real sense.
\end{abstract}

\section{Keywords- Virtual Building; VRML; Virtual Reality}

\section{INTRODUCTION}

Virtual reality technology (VR) is the new technology appearred in recent years.It is an environment which is produced by a computer and seems very like reality to the person experiencing it. It can provide users with many kinds of simulation such as visual sense,auditory sense and touch reception so that people can observe all the sights without restrictions. The technology of virtual walkthrough is an important branch of virtual reality technology.It develops rapidly in the architecture,tourism,game industry, aerospace, medicine and other industries. It has three characteristics: immersion, interaction and imagination and we call them "3I". When users enter into the vitual environment by all kinds of advanced sensors, they think all of the things they see,hear and feel are real and they believe they are in the real environment, that is called "immersion". Interaction means that all users can interact with many objects in the vitual environment and imagination means users can have many association with the immersion and interaction in the vitual environment ${ }^{[1]}$.

\section{DEVELOPMENT TECHNOLOGY}

\section{A. Development Method}

In order to improve the immersion, interaction and imagination of the virtual system, we use the VRML and 3D modelling technologies in this roamming system.We also apply the improved AABB bounding box algorithm to test the collision.

\section{B. VRML Nodes}

VRML (Virtual Reality Modeling Language) is a virtual reality modeling language. It is a popular language to develope three-dimensional website based on www on internet and it is independent with platform.VRML is a kind of interpreted language whose objects are nodes.All of complex scenes are made of nodes.

The main nodes of VRML are shown as follow ${ }^{[2]}$ :

1)Modelling nodes. There are many geometry,group and location nodes in VRML which can model the object easily.

(1) Basic geometrical node: Box, Sphere, Cone, Cylinder;

(2) Constructed geometrical node: IndexLineSet, IndexFaceSet, Extrusion, PiontSet, ElevationGrid;

(3) Location and rotation node: Group, Transform;

(4)Special shape node: Billbord, Backgroud, Text.

Basic geometrical node can only make some limited objects. Constructed geometrical node can make complex point,line,surface and other objects. Location and rotation node can describe the assemble location and direction. Special shape node can set the text and background color in the scene.

2). Rendering nodes

(1)Light node:HeadLight, SpotLight,PointLight,DirectionLight;

(2)Material and color node:Material, Appearance, Color, ColorInterpolator;

(3)Texture node: ImageTexture, MoviceTexture, PixelTexture, TextureTransform;

(4)Other nodes:Fog, Normal,NormalInterpolator, Sound.

These light nodes can provide the light source for the virtual scene which can decide users' visual effect. Material and color node can make the objects more real. Sound node can simulate all kinds of sound.

3).Observation and interaction nodes

(1)Sensor:CylinderSensor,PlaneSensor, VisibilitySensor, ProxymitySensor,SphereSensor,TouchSensor;

(2)Control viewpoint: ViewPoint, NavigationInfo.

Each sensor can sense the mouse pointer of the user and the viewpoint can provide user a better perspective.

4).Animation nodes

(1)Frame time sensor: TimeSensor;

(2)Posture adjustment: CoordinateInterpolator, OrientationInterpolator, ScalarInterpolator.

VRML provides a convenient way of animation control.The two group of nodes can be used to produce the scene animation.

5).Level of detail management and collision detection nodes: LOD,Collision. Level of detail management can 
control the detail display of complex entity. Collision detection can detect the colllision of motorial objects.

6.Hyperlinks and Embedding nodes:Anchor, Inline.

The two nodes can link one scene with another or embed one scene in another.

\section{VRMLPad}

VrmlPad is one of the most powerful develop tools which was developed by Parallel Graphics corporation.It is based on text format and real-time preview.It can download, edit, preview and debug the VRML code.

The great advantage of Vrmlpad is that it can automatically identify the users' input type in VRML and find the possible errors. It can combine with browser and provide many plug-in unit.So it is the most popular compiler in VRML. It could not only support the grammar tips, syntax coloring, bookmarks and other settings, but also the structure of scene graph tree, routing plans and resources graph and so on, thus could quickly query and orienting; in addition, it also provides a preview function, in which you can immediately see the effect of 3D scene rendering. It reduces the quantity of input process, and greatly enhanced the efficiency of the development.

\section{SYSTEM DESIGN}

\section{A. System Design}

The flow chart of Fragrance Hill roamming system is shown as follow ${ }^{[3]}$ :

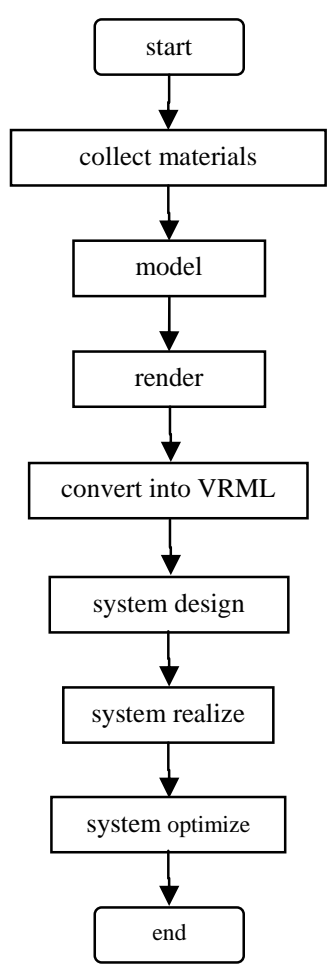

Figure 1. The system design process

\section{B. Design Process}

1).Collect materials. Collecting materials is selecting and sampling the modeling object. In this paper, we take Beijing Fragrant Hills Park as example to realize the system roamming.Fragrant Hill is one of the famous feature spots in Beijing. The buildings are Chinese ancient royal architecture, which has Chinese architectural style.

a)Collection method. In this system,we adopts the way for photo collection. The photo collection can convert the digital images into materials easily. Figure 2 is the picture of the main hall.

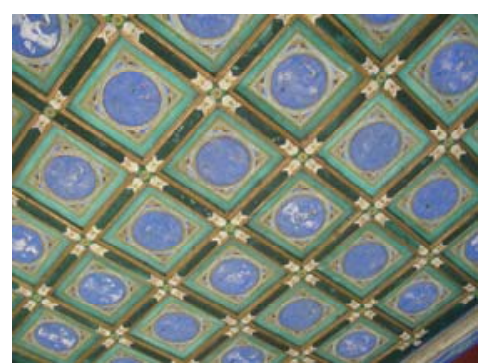

Figure 2. THE PICTURE OF THE MAIN HALL

b).Material fabrication. We can apply the collected pictures into the software to make materials such as Photoshop.

2).Model System. 3DSMAX is the world's most popular animation modeling software and its function of outputting the three-dimensional model into VRML format makes it the best assistant of VRML.

Three-dimensional modeling should be technically divided into three steps:

a)geometric modeling.The presentation and manipulation of object geometric information is the study object of Geometric modeling technology. It refers to the data structure indicating geometric information, as well as relating structures and algorithms which manipulate this data structure. Geometric modeling can be further divided into hiberarchy modeling and category modeling ${ }^{[4]}$.

b)physical modeling. Collision Detection is a commonly used method; it is the detection process of whether virtual objects have had collision in the virtual space. The fundamental principle of Collision detection technology is that if two completely sealed polyhedrons come into collision, at least one surface of one polyhedron intersects with at least one surface of another one. A suitable response that modifying the physical properties of virtual objects should be made when a collision is detected ${ }^{[5]}$.

c)Behavioral Modeling.Behavioral Modeling is to endow the model inherent characteristics or properties as soon as they are created ${ }^{[6]}$.

3). Render. Rendering refers to the software process converting from models to images. The model is the description for 3D objects with strictly defined language or 
data structure,including geometry, viewpoint, texture and lighting information. There are two kinds of images: digital image or bitmap image.

Rendering is applied for many fields such as computer and video games, simulation, movies or TV animation and visualization design. As a product of view, rendering now has a variety of tools products, some integrate into larger modeling or animation package, some are independent products, some are open source products. From the internal perspective, rendering tools are designed carefully based on various theories,including optics, visual perception, mathematics and software development.

4). Convert into VRML. The 3Dmax can derive .wrl file which is the VRML format. Because the position and the physical form are different from VRML,the entities converted from 3Dmax are some nodes.

Because the VRML language itself has the concept of hierarchical levels, including group and children.Various geometric elements in VRML scene can form a tree. The main scene structure is shown in Figure 3.

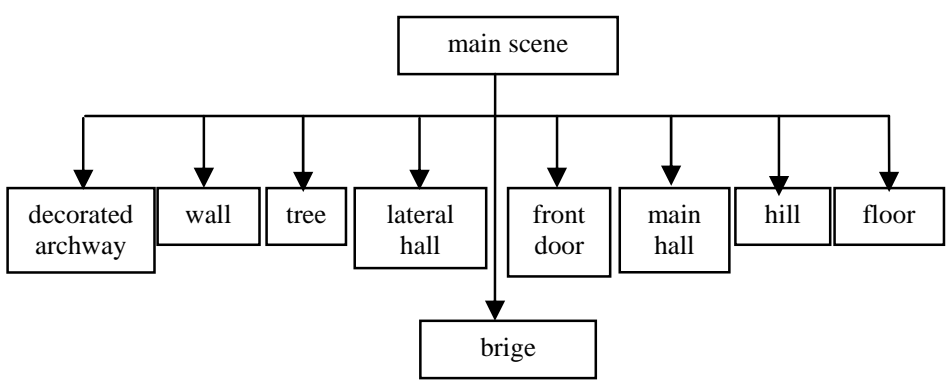

Figure 3. THE MAIN SCENE STRUCTURE

The nodes used in this system include:

(1) shape node

DEF environment Group \{

children [

Shape \{

appearance Appearance \{

material Material $\{\ldots\}$

texture ImageTexture $\{\ldots\}$

textureTransform TextureTransform $\{\ldots\}$

geometry DEF shui IndexedFaceSet \{

color Color $\{$ color $[\ldots]\}$

colorIndex $[\ldots]$

coord DEF shui-COORD Coordinate $\{$ point $[\ldots]\}$

coordIndex $[. .$.

\}

\}

]

These codes are generated by converting the 3D graphics.We can describe the entity based on each point coordinates, color and direction.But follow codes are drawing basic graphic.

DEF environment Group \{

children [
Shape \{

appearance Appearance \{

material Material $\{\ldots\}$

texture ImageTexture $\{\ldots\}$

textureTransform TextureTransform $\{\ldots\}$

geometry box $\{$ size 0000$\}$

\}

\}

]

(2) Inline node

Because the tree and mountain files are very small and repeated more frequently, the use of inline node can reduces the file size.The structure of inline node is:

DEF shan Transform \{

children [Inline \{\} ]

\}

5. System realize

(1) Collision Detection

NavigationInfo \{

avatarSize [0.1 1.5 1.5]

speed 2

type "ANY"

Transform \{

children DEF PS ProximitySensor \{

center 000

size 100010001000

\}

(2) Background

Background \{

skyColor [

0.00 .20 .7

0.00 .51 .0 ,

1.01 .01 .0

skyAngle [ 1.309, 1.571 ]

frontUrl "pic/sky.jpg"

backUrl "pic/sky.jpg"

leftUrl "pic/sky.jpg"

rightUrl "pic/sky.jpg"

\}

(3) Sunlight

DEF sunlight PointLight \{

intensity 0.5

location 0600600

radius 1000

\}

(4) Autorotation

Transform \{

translation 00.00 .0

children [

DEF Planter Transform \{

scale 0.50 .50 .5

children [

Shape \{

appearance Appearance \{

material Material \{

diffuseColor .61 .61 .61 
specularColor .77 .76 .76

ambientIntensity .14

shininess .9

\}\}

geometry Box \{

size 2.52$\}$

\}

]

6.VRML file optimization

The optimization methods include:

(1)The reuse mechanism of object.

(2)The use of inline node.

(3)Compression of VRML file.

7.The system interface

The system interface is shown as follow:

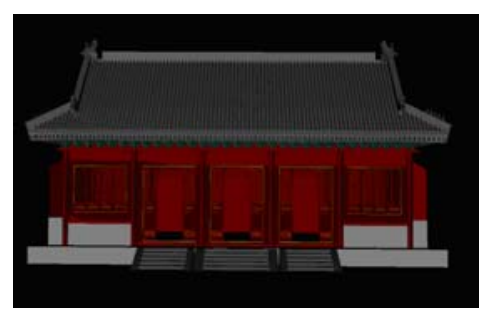

Figure 4 The main hall

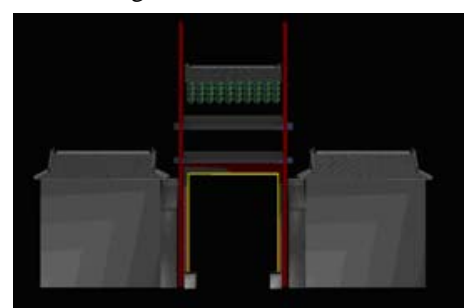

Figure 5 The decorated archway

\section{SUMMARY}

VRML is a powerful tool to provide a better mode to build lifelike three-dimensional scenes.It is applied widely in many fields such as architecture, tourism, film and television industry and so on. This system take the Fragrant Hill Park as the background and realize the vitual roamming system.The system result shows that it is real-time and authentic. It can provide the users immersion, interaction and imagination.

\section{REFERENCES}

[1] Zhang Zhengxia,Wang Houxiang, An Outline and Prosprect of VRML, Journal of Engineering Graphics:1998(2):85-90

[2] HE Lai-kun, XU Yuan, Introduction and appl ication of Virtual Reality Modeling Language, Journal of Hangzhou Teachers College( Natural Science Edition):2005(4):157-160

[3] Na Shun,The investigation of VRMLprinciple and application, Journal of Inner Mongolia University for Nationalities,2009(15):1718

[4] Song Huiling, Research and Implementation of Virtual Geology Museum Based on VRML, Master's degree thesis of China University of Geosciences: 2004

[5] Wand Xiaorong,Wang Meng,Li Chungui.Research of Collision Detection Algorithms Based on AABB.Computer Engineering \& Science.2010(32):59-61

[6] Zhou Jian,Gao Wen,Zhao Xinmin, Models for Constructing Moving Object in Virtual Reality.Journal of Harbin Institute of Technology.1997(2):40-43

\footnotetext{
${ }^{\mathrm{i}}$ This work is supported by 2012 science and technology project of Beijing Municipal Commission of Education, the number is 051200315.
} 